\title{
Foot and Ankle
}

LETTER TO THE EDITOR

\section{Freiberg Disease Presenting as Forefoot Pain}

\author{
Ferit Dogan ${ }^{1}$, Mustafa Turgut Yildizgoren ${ }^{2 *}$ and Pelin Zeynep Bekin Sarikaya ${ }^{1}$ \\ ${ }^{1}$ Department of Radiology, Balikligol State Hospital, Şanlıurfa, Turkey \\ ${ }^{2}$ Department of PM\&R, Medical School, Mustafa Kemal University, Hatay, Turkey
}

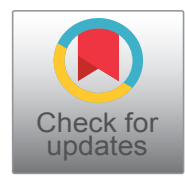

*Corresponding author: Mustafa Turgut Yildizgoren, Department of PM\&R, Medical School, Mustafa Kemal University, Hatay, Turkey, Tel: +903262291000, Fax: +903262295654, E-mail: ftr.mustafaturgut@hotmail.com

\section{Keywords \\ Forefoot, Pain, Freiberg disease}

To the editor,

A 19-year-old female presented with a 3-month history of pain along the dorsal aspect of the right forefoot. She had permanent footpain, which worsened when walking. No history of trauma was noted. Physical examination revealed evident swelling and tenderness near the second Metatarsophalangeal Joint (MTJ). An X-ray of the right foot revealed sub- chondral sclerosis of the second metatarsal head with fragmentation within the joint (Figure 1A). In addition, Magnetic Resonance Imaging (MRI) demonstrated hypointense signals on T1-weighted images and increased signal intensity on T2-weighted images (Figure 1B and Figure 1C). In the light of the clinical and radiological information, Freiberg's disease was diagnosed. Initially the patient was treated with nonsteroidal anti-inflammatory medications, activity modification and, metatarsal pad. After 2 months of treatment the patient obtained pain relief, and she was walking pain-free.
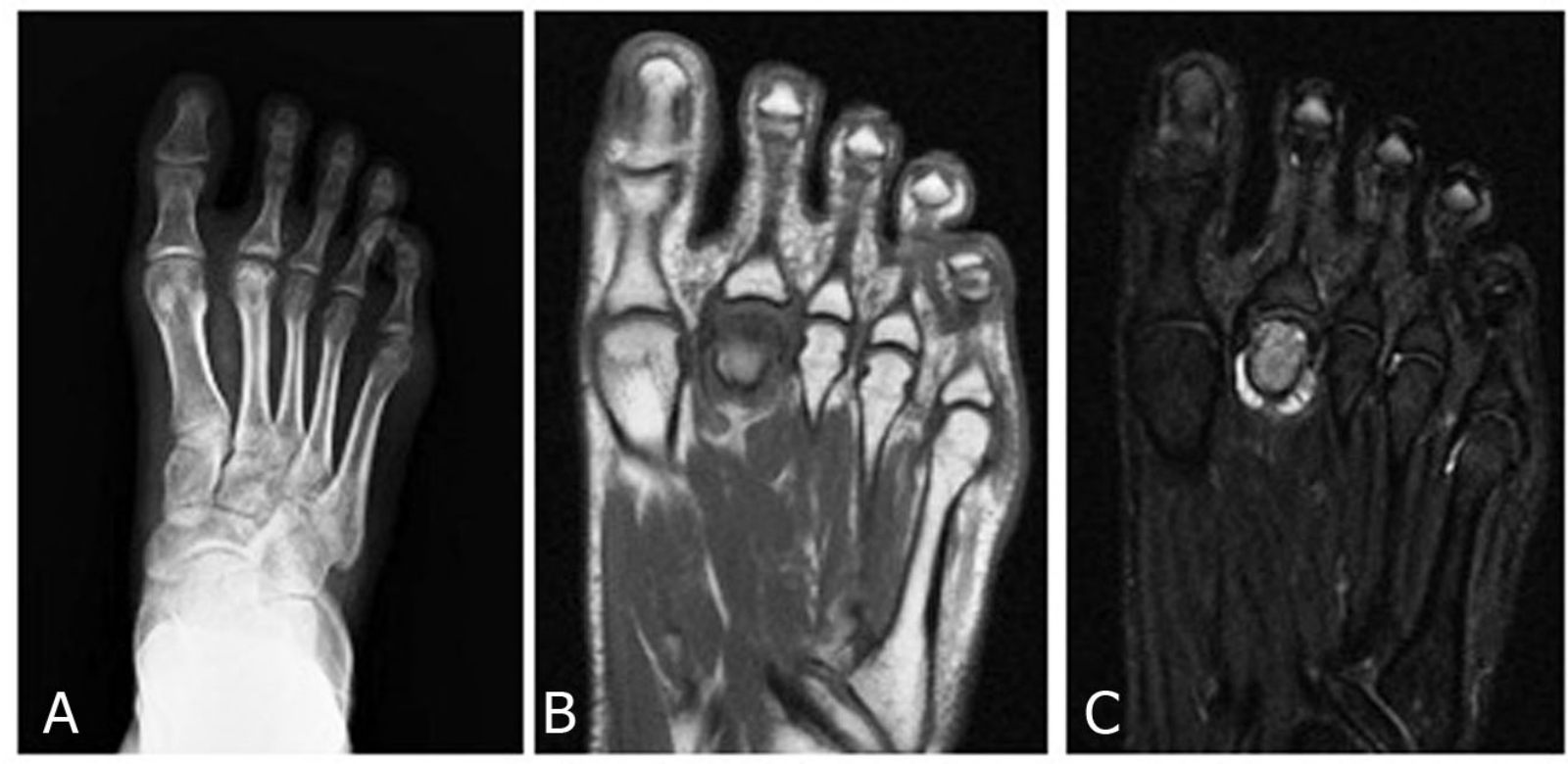

Figure 1: Antero-posterior radiograph of the right foot showing flattening of the second metatarsal head and subchondral sclerosis with fragmentation within the metatarsophalangeal joint (A) T1-weighted (B) and T2-weighted (C) Coronal MRI views of the rightfoot showing hypointense signals and hyperintense signals at the second metatarsal head, respectively.

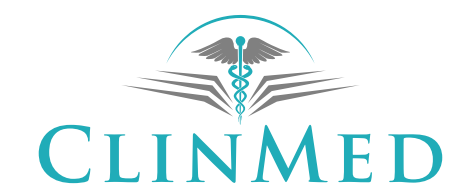

INTERNATIONAL LIBRARY
Citation: Dogan F, Yildizgoren MT, Sarikaya PZB (2017) Freiberg Disease Presenting as Forefoot Pain. Int J Foot Ankle 1:004.

Received: August 12, 2017; Accepted: December 14, 2017; Published: December 16, 2017

Copyright: (c) 2017 Dogan F, et al. This is an open-access article distributed under the terms of the Creative Commons Attribution License, which permits unrestricted use, distribution, and reproduction in any medium, provided the original author and source are credited. 
Freiberg's disease is typically described as a vascular necrosis of a metatarsal head. It most commonly affects the second metatarsal. Bilateral involvement has been published in the literature [1]. The classical location of this disorder is the second MT head (68\%), although it can also be found in the other lesser MTs, most commonly the third $(27 \%)$, followed by the fourth (3\%). The fifth MT head is rarely involved. Cases of multiple and bilateral MT involvement have also been reported [2]. With a multifactorial etiology including mechanical stress, repetitive trauma and vascular dysfunction, the condition is five times more common in females than males, especially in adolescents [3]. Patients often present with pain and swelling around the MTJ [2]. Although, plain radiographs are generally used to diagnose Freiberg's disease, there are no radiographic changes in the early stages. Moreover, MRI examinations are useful in the diagnosis of Freiberg's disease, especially in the early stage of the disease. The typical radiographic appearance is of flattening of the second metatarsal head, increased sclerosis and loose body formation within the joint. MRI is useful in diagnosis, as T2-weighted images often show increased signal intensity in the metatarsal head [4]. The differential diagnosis for Freiberg's disease includes stress fracture, tendonitis, morton neuroma and metatarsalgia. Since most cases are self-limited, conservative treatment including activity modification, insoles, metatarsal pads, casting, or controlled ankle motion and non-steroidal anti-inflammatory medications may be adequate. Con- servative treatment consists of immobilization with or without crutches for 4 to 6 weeks or until symptoms resolve. In the literature, conservative treatment and preventive treatment decrease a symptoms at 4 to 6 weeks. Rarely indicated and reserved for patients who do not respond to conservative management, surgical treatment can range from metatarsal neck osteotomy and joint debridement to resection of the metatarsal head. Abnormal stress associated with chronic traumatic insult may explain the occurrence of osteonecrosis of the second metatarsal bone in our patient.

\section{Conflict of Interest}

The authors declare that they have no conflict of interest.

\section{Acknowledgement}

None.

\section{References}

1. Lui TH (2015) Thompson and Hamilton type IV Freiberg's disease with involvement of multiple epiphyses of both feet. BMJ Case Rep.

2. Waldman SD, Campbell RSD (2011) Freiberg's Disease. In: Imaging of Pain. (1 ${ }^{\text {st }}$ edn), Philadelphia, PA, Saunders, 447-448.

3. Cerrato RA (2011) Freiberg's disease. Foot Ankle Clin 16: 647-658.

4. Carmont MR, Rees RJ, Blundell CM (2009) Current concepts review: Freiberg's disease. Foot Ankle Int 30: 167-176. 